\title{
Automated detection and quantification of COVID-19 pneumonia: CT imaging analysis by a deep learning-based software
}

\author{
Hai-tao Zhang ${ }^{1,2} \cdot$ Jin-song Zhang ${ }^{2,3} \cdot$ Hai-hua Zhang ${ }^{1} \cdot$ Yan-dong Nan ${ }^{1,2} \cdot$ Ying Zhao ${ }^{2,4} \cdot$ En-qing Fu $^{1,2}$. \\ Yong-hong Xie ${ }^{1,2} \cdot$ Wei Liu ${ }^{1,2} \cdot$ Wang-ping $\mathrm{Li}^{1,2} \cdot$ Hong-jun Zhang ${ }^{1,2} \cdot$ Hua Jiang ${ }^{1,2} \cdot$ Chun-mei Li $^{1,2} \cdot \mathrm{Yan}^{1}$-yan $\mathrm{Li}^{1,2} \cdot$ \\ Rui-na Ma $^{1,2} \cdot$ Shao-kang Dang ${ }^{1,2} \cdot$ Bo-bo Gao ${ }^{1,2} \cdot$ Xi-jing Zhang ${ }^{2,5} \cdot$ Tao Zhang ${ }^{1,2}$ (D)
}

Received: 30 March 2020 / Accepted: 5 July 2020 / Published online: 14 July 2020

(C) Springer-Verlag GmbH Germany, part of Springer Nature 2020

\begin{abstract}
Background The novel coronavirus disease 2019 (COVID-19) is an emerging worldwide threat to public health. While chest computed tomography (CT) plays an indispensable role in its diagnosis, the quantification and localization of lesions cannot be accurately assessed manually. We employed deep learning-based software to aid in detection, localization and quantification of COVID-19 pneumonia.

Methods A total of 2460 RT-PCR tested SARS-CoV-2-positive patients (1250 men and 1210 women; mean age, 57.7 \pm 14.0 years (age range, 11-93 years) were retrospectively identified from Huoshenshan Hospital in Wuhan from February 11 to March 16, 2020. Basic clinical characteristics were reviewed. The uAI Intelligent Assistant Analysis System was used to assess the CT scans.

Results CT scans of 2215 patients (90\%) showed multiple lesions of which $36(1 \%)$ and 50 patients (2\%) had left and right lung infections, respectively ( $>50 \%$ of each affected lung's volume), while $27(1 \%)$ had total lung infection (>50\% of the total volume of both lungs). Overall, $298(12 \%), 778(32 \%)$ and $1300(53 \%)$ patients exhibited pure ground glass opacities (GGOs), GGOs with sub-solid lesions and GGOs with both sub-solid and solid lesions, respectively. Moreover, 2305 (94\%) and 71 (3\%) patients presented primarily with GGOs and sub-solid lesions, respectively. Elderly patients ( $\geq 60$ years) were more likely to exhibit sub-solid lesions. The generalized linear mixed model showed that the dorsal segment of the right lower lobe was the favoured site of COVID-19 pneumonia.

Conclusion Chest CT combined with analysis by the uAI Intelligent Assistant Analysis System can accurately evaluate pneumonia in COVID-19 patients.
\end{abstract}

Keywords 2019 novel coronavirus · Viral pneumonia · Artificial intelligence (AI) · Computed tomography (CT) · Ground glass opacity (GGO)

Hai-tao Zhang, Jin-song Zhang and Hai-hua Zhang contributed equally to this work.

This article is part of the Topical Collection on Advanced Image Analyses (Radiomics and Artificial Intelligence)

Xi-jing Zhang

zhangxj918@163.com

Tao Zhang

zhangft@fmmu.edu.cn

1 Department of Pulmonary and Critical Care Medicine, Tangdu Hospital, Air Force Military Medical University, Xi'an 710038, China
Wuhan Huoshenshan Hospital, Wuhan 430100, China

3 Department of Radiology, Xijing Hospital, Air Force Military Medical University, Xi'an 710038, China

4 Tangdu Hospital, Air Force Military Medical University, Xi'an 710038, China

5 Department of Critical Care Medicine, Xijing Hospital, Air Force Military Medical University, Xi'an 710038, China 


\section{Introduction}

In December 2019, an outbreak of a disease was reported in Wuhan, Hubei province, China, with a novel coronavirus, called Severe Acute Respiratory Syndrome Coronavirus 2 (SARS-CoV-2) as its cause [1]. The World Health Organization (WHO) had then named the disease caused by the novel coronavirus as Coronavirus Disease 2019 (COVID19). On March 11, 2020, the WHO declared COVID-19 to be a pandemic [2], with 10,021,401 confirmed cases and 499,913 reported deaths globally, as of June 29, 2020 [3].

At present, real-time reverse-transcription-polymerasechain-reaction (real-time RT-PCR) is the standard method used to make a definitive diagnosis of SARS-CoV-2 infection [4]. Unfortunately, RT-PCR results can be affected by sampling errors and low virus load [5,6]. Radiological examinations, especially thin slice chest Computed tomography (CT) scans, play an important role in identifying the early phase of lung infection, monitoring disease progression and guiding clinical decision making for COVID-19 patients [5, 7, 8]. However, radiologists in different countries vary in their abilities to distinguish COVID-19 from other viral pneumonias [9].

Due to the low contrast of the infection regions in CT images and large variations in both shapes and positions of lesions in different patients, delineation of infection regions in chest $\mathrm{CT}$ scans is very challenging. Li et al. had reported initial experiences of artificial intelligence (AI)-based tools to diagnose COVID-19 pneumonia on radiological images [10]. However, the quantification and localization of lesions analysed by AI software were not addressed. In the present study, the uAI Intelligent Assistant Analysis System, a deep learning-based software, was used to automatically extract and analyse regions suspected to be infected with the virus. The distribution and volume of infection in the lungs, as well as the CT features of COVID-19, were assessed.

\section{Methods}

\section{Patient cohort}

RT-PCR tested SARS-CoV-2-positive patients who were retrospectively identified from Huoshenshan hospitals in Hubei Providence, China, from February 11 to March 16, 2020. The RT-PCR results were extracted from the patients' electronic medical records in the hospital information system. The clinical data analysed were as follows: age, sex, comorbid conditions and symptoms. Altogether, 2460 patients (mean age, $57.7 \pm 14.0$ years; range: $11-93$ years) with COVID-19 were included in the study of which 1250 patients $(51 \%)$ were male (mean age: $57.2 \pm 14.4$ years; range: $14-91$ years) and 1210 $(49 \%)$ were female (mean age: $58.3 \pm 13.6$ years; range; $11-$ 93 years) (Table 1). The ethics committee at Wuhan
Huoshenshan Hospital waived the need for informed consent for this retrospective study.

\section{CT scanning protocol}

Multiple detector computed tomography (MDCT) was performed using the uCT 760 scanner (United Imaging, Shanghai, China). The parameters used for the scanning protocol were as follows: patient in the supine position; end inspiratory acquisition; tube voltage, $120 \mathrm{kV}$; tube current-exposure time product, $145 \mathrm{mAs}$; section thickness, $0.625 \mathrm{~mm}$; section thickness after reconstruction, $10 \mathrm{~mm}$; and spacing $10 \mathrm{~mm}$. Unenhanced CT scans were obtained for all patients.

\section{Quantification and assessment of COVID-19 infection by uAl}

The uAI Intelligent Assistant Analysis System, a deep learningbased software, was specifically developed by United Imaging Medical Technology Company Limited (Shanghai, China) for COVID-19 assessment. This AI software has a modified 3D convolution neural network and a combined V-Net with bottleneck structures [11,12]. Detailed information on the same was provided in previous reports $[13,14]$. The lung segmentation model was launched as early as 2018 , while latest models such as infection segmentation and lung field segmentation were added in Feb-Mar, 2020. As detailed in previous report [13], 249 and 300 CT images from COVID-19 patients were used for training and evaluation, respectively. A deep learning-based segmentation network called VB-Net was proposed, which achieved $91 \%$ overlap with manual segmentations for infections, and $98 \%$ overlap for the lung region. The population in this work is different from the previous datasets used for training and evaluation.

The baseline CT scans of patients were first fed to the deep learning-based segmentation system, which generated infection areas in the whole lung, lung lobes and all bronchopulmonary segments. After segmentation, various metrics were computed to quantify the COVID-19 infection, including volumes of infection in the whole lung, lung lobes and bronchopulmonary segments. Two radiologists made the necessary manual changes for identifying the atypical lesions to validate the results.

In addition, the percentages of infection in the whole lung, lung lobes and bronchopulmonary segments were also computed to measure the severity of the disease and to describe the anatomical pattern within the lung. The Hounsfield unit (HU) histogram within the infection region can be visualized for evaluation of ground glass opacity (GGO) and solid components inside the infection region. The intelligent software used the HU value to classify the lesion into three types: less than 300 for GGO, -300 to 50 for sub-solid and greater than 50 for solid. 
Table 1 Patient characteristics

\begin{tabular}{ll}
\hline Characteristic & Value \\
\hline Age (years, mean \pm SD) & $55.7 \pm 14.9$ \\
Male & $1250(51 \%)$ \\
Range (10-29) & $51(2 \%)$ \\
Range (30-49) & $306(12 \%)$ \\
Range (50-69) & $647(26 \%)$ \\
Range (70-100) & $246(10 \%)$ \\
Female & $1210(49 \%)$ \\
Range (10-29) & $38(2 \%)$ \\
Range (30-49) & $232(9 \%)$ \\
Range (50-69) & $722(29 \%)$ \\
Range (70-100) & $218(9 \%)$ \\
Comorbidities & \\
Hypertension & $543(22 \%)$ \\
Diabetes & $255(10 \%)$ \\
Cardiovascular disease & $94(4 \%)$ \\
Liver disease & $51(2 \%)$ \\
Nervous system disease & $44(2 \%)$ \\
Chronic lung disease & $50(2 \%)$ \\
Chronic kidney disease & $26(1 \%)$ \\
Tumour & $32(1 \%)$ \\
Self-reported symptoms & \\
Fever & $1807(73 \%)$ \\
Cough & $1734(70 \%)$ \\
Fatigue & $1388(56 \%)$ \\
Dyspnoea & $1194(49 \%)$ \\
Myalgia & $791(32 \%)$ \\
Productive cough & $391(16 \%)$ \\
Diarrhoea & $153(6 \%)$ \\
Nausea or vomiting & $86(3 \%)$ \\
Headache & $77(3 \%)$ \\
Outcome & \\
Recovery & \\
Death & \\
\hline & \\
\hline
\end{tabular}

Data presented as $n(\%)$ unless otherwise indicated

\section{Statistical analysis}

All statistical analyses were performed using SPSS (IBM Corp., Armonk, NY, USA). Continuous variables are expressed as mean $\pm \mathrm{SD}$. Categorical variables are expressed as counts and percentages. The Chi-square test was used to compare the distribution of $\mathrm{CT}$ features in relation to age and sex. The generalized linear mixed model was used to compare the incidence of infection in different lung segments. In this model, lung segment, age and sex were used as fixed effects, and patients were used as random effects. The odds ratios (OR) and 95\% confidence intervals $(\mathrm{CI})$ are reported.

\section{Results}

Table 1 summarizes the patients' clinical characteristics. The common patient comorbidities included hypertension, diabetes, coronary atherosclerotic heart disease, liver disease, nervous system disease, chronic lung disease, chronic kidney disease and tumour. The common self-reported symptoms of illness were fever $(73 \%)$, cough $(70 \%)$, fatigue $(56 \%)$, dyspnoea (49\%), myalgia (32\%) and productive cough (16\%). As of April 10, 2447 (99\%), patients had recovered and $13(1 \%)$ patients had died.

Figure 1 shows the complete pipeline for quantitative COVID-19 assessment. Lesions on chest CT were automatically identified by the intelligent software. Only a few atypical lesions required manual identification (Fig. 2).

Among the $2460 \mathrm{CT}$ images, 2215 (90\%) showed manifestations of COVID-19 pneumonia in both the lungs. Altogether, 167 (7\%) showed unilateral pulmonary infection with $81(3 \%)$ in the right lung and 86 (3\%) in the left, while 84 patients $(3 \%)$ had negative chest CT scans. Altogether, $36(1 \%)$ and $50(2 \%)$ patients had left and right lung infection, respectively, accounting for more than $50 \%$ of each affected lung volume, and $27(1 \%)$ patients had total lung infection accounting for more than $50 \%$ of the total volume of both lungs (Table 2).

In terms of lesion distribution, infection was found in the apicoposterior segment of the left upper lobe, the anterior segment of the left upper lobe, the superior lingular segment of the left upper lobe, the inferior lingular segment of the left upper lobe, the dorsal segment of the left lower lobe, the anterior medial basal segment of the left lower lobe, the lateral basal segment of the left lower lobe, the posterior basal segment of the left lower lobe, the apical segment of the right upper lobe, the posterior segment of the right upper lobe, the anterior segment of the right upper lobe, the lateral segment of the right middle lobe, the medial segment of the right middle lobe, the dorsal segment of the right lower lobe, the medial basal segment of the right lower lobe, the anterior basal segment of the right lower lobe, the lateral segment of the right lower lobe and the posterior basal segment of the right lower lobe in $1756(71 \%), 1624(66 \%), 1603(65 \%), 1575(64 \%)$, 1797 (73\%), 1696 (69\%), 1813 (74\%), $1884(77 \%), 1501$ (61\%), 1802 (73\%), 1630 (66\%), $1548(63 \%), 1482(60 \%)$, 1958 (80\%), 1225 (50\%), 1766 (72\%), 1837 (75\%) and 1892 (77\%) cases of 2460 cases, respectively (Fig. 3). The generalized linear mixed model showed that the favoured site of COVID-19 pneumonia was the dorsal segment of the right lower lobe $(p<0.001)$ (Table 3).

In terms of lung changes, the appearance of different types of lung is shown in Fig. 4. CT scans showed that $298(12 \%), 778(32 \%)$ and $1300(53 \%)$ patients had pure GGO, GGO with sub-solid lesions and all three types, 

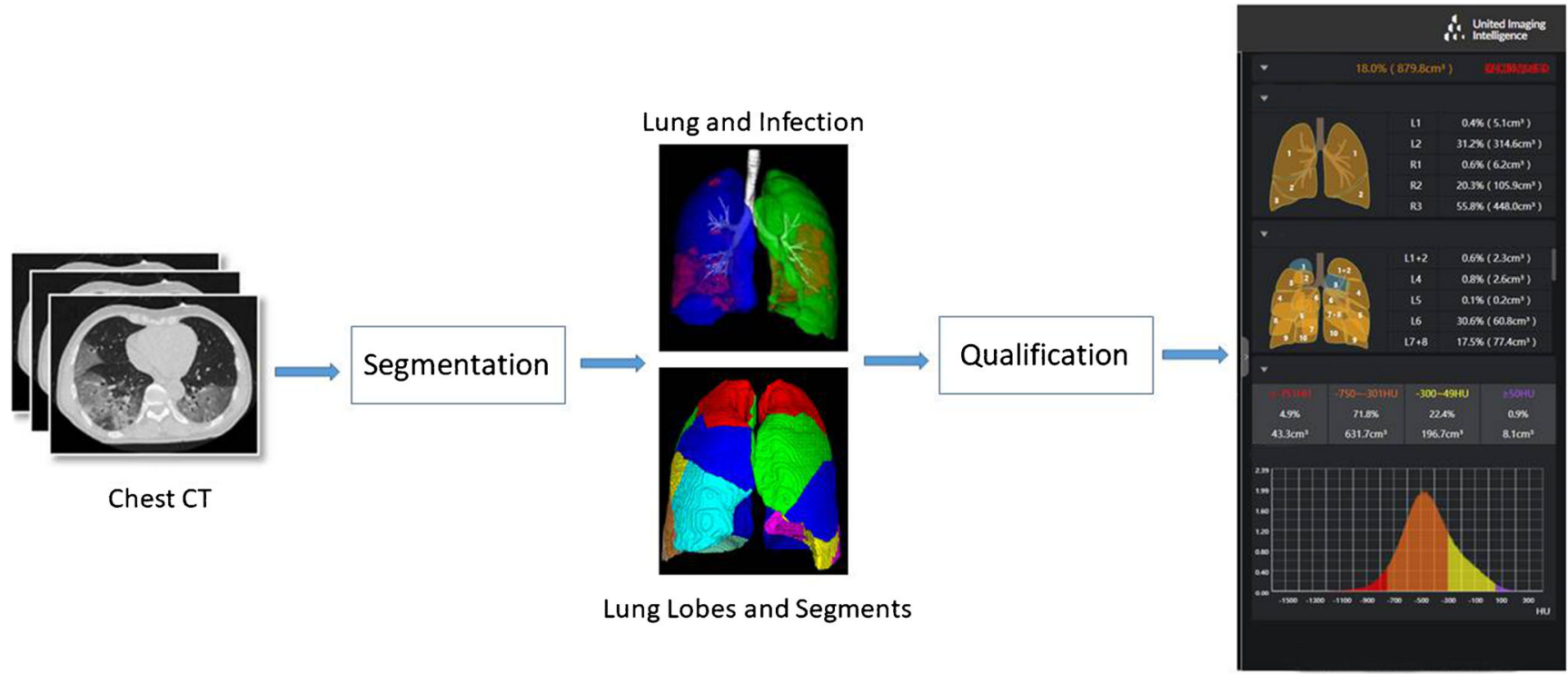

Analysis Result

Fig. 1 Pipeline for quantifying COVID-19 infection A chest CT scan is first fed into the deep learning-based segmentation system. Then, quantitative metrics are calculated to characterize infection regions in the CT

respectively. There were 2305 patients (94\%) and 71 patients (3\%) primarily presenting with GGO and subsolid lesions, respectively (Table 2). As demonstrated in Table 3, the distribution of CT scan signs was not significantly different between male or female patients ( $p=$ 0.4125 ). However, elderly patients ( $\geq 60$ years) were more likely to develop pneumonia and manifest with sub-solid lesions in CT scans compared to younger patients $(p<0.001)$ (Table 4). scan, including but not limited to the following: infection volumes, and percentages of infection (POIs) in the whole lung, lung lobes, and bronchopulmonary segments

\section{Discussion}

Recently, COVID-19 cases have been escalating worldwide. This has led to an increasing use of radiological examinations, such as the chest CT, to aid in diagnosis, determination of patient prognosis and evaluation of the COVID-19 disease course by physicians. Li et al. had reported that AI software could accurately detect and differentiate COVID-19 pneumonia from community-acquired pneumonia and other lung

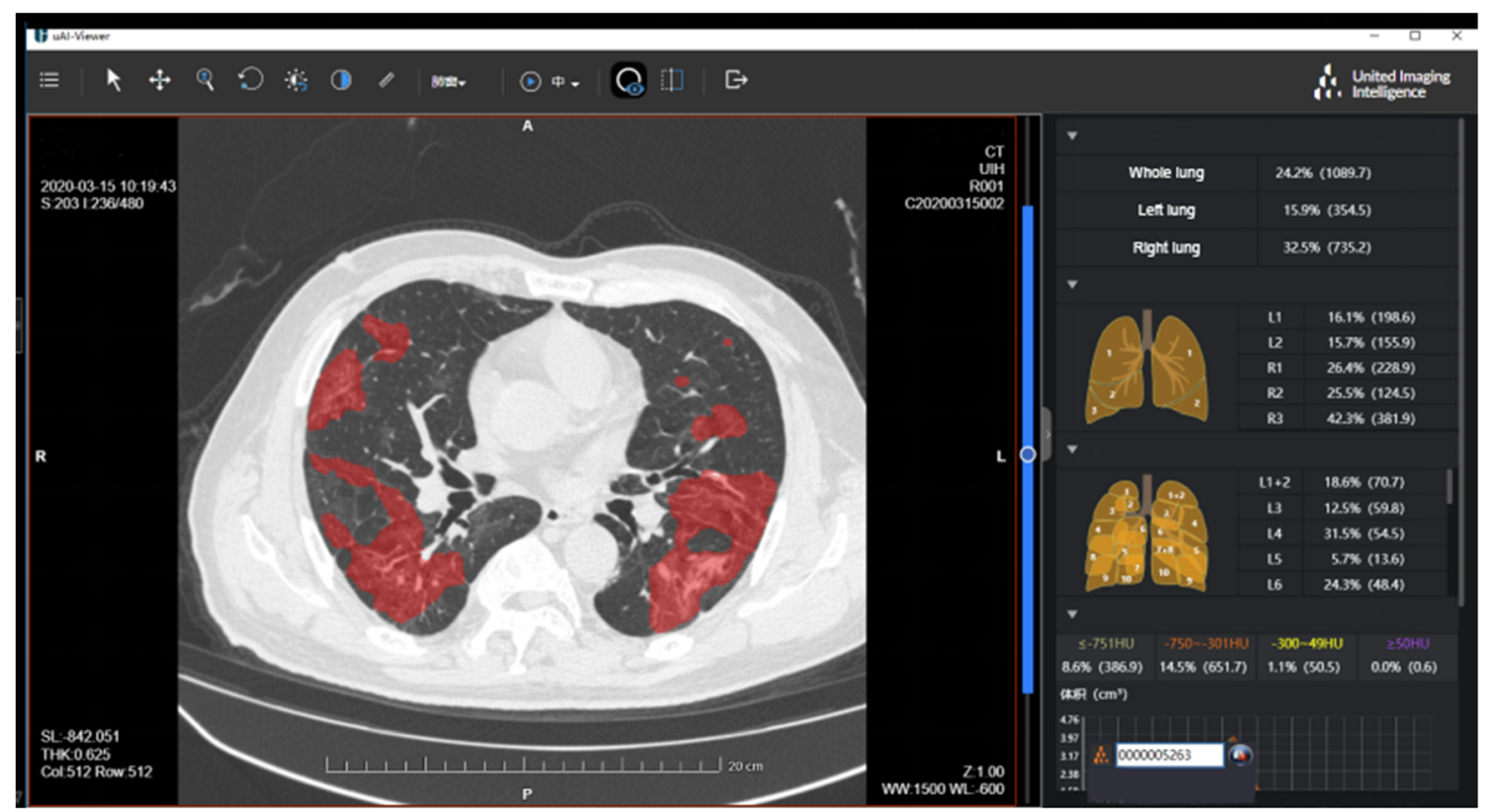

Fig. 2 Representative uAI output of a 54-year-old man with COVID-19 CT images showing bilateral lesions, which were analysed by the uAI intelligent assistant system 
Table 2 Chest CT features of patients with COVID-19

\begin{tabular}{ll}
\hline CT feature & No. (\%) of patients \\
\hline Lesion presentation on scan & \\
Negative & $84(3 \%)$ \\
Unilateral infection & $167(7 \%)$ \\
Only right lung & $81(3 \%)$ \\
Only left lung & $86(3 \%)$ \\
Bilateral infection & $2215(90 \%)$ \\
Left lung infection volume greater than 50\% & $36(1 \%)$ \\
Right lung infection volume greater than 50\% & $50(2 \%)$ \\
Total lung infection volume greater than 50\% & $27(1 \%)$ \\
CT signs & \\
Pure GGO & $298(12 \%)$ \\
GGO + sub-solid & $778(32 \%)$ \\
GGO + sub-solid + solid & $1300(53 \%)$ \\
GGO as the main lesion & $2305(94 \%)$ \\
Sub-solid as the main lesion & $71(3 \%)$ \\
\hline
\end{tabular}

$C T$ computed tomography, GGO ground glass opacity

diseases [10]. However, the quantitative assessment of CT features by AI software has not been reported extensively. These objective data are urgently needed by clinical workers $[14,15]$. In this study, we analysed the CT scans of 2460 COVID-19 patients using the uAI Intelligent Assistant Analysis System. This software can accurately segment different anatomical structures of the lung to detect infection regions and measure the percentage of infection in both lungs, along with its lobes and segments. The measures can be adjusted using customized HU ranges.

It has been reported that patients affected by COVID-19 pneumonia show bilateral and multilobar involvement in CT scans, with lesions being more frequent in the lower lobes [16,
17]. Consistent with these studies, our data also showed that COVID-19 pneumonia manifested in both lungs in 2215 of 2460 patients. Furthermore, the study found that the favoured site of COVID-19 pneumonia is the dorsal segment of the right lower lobe. This may be because of the innate anatomic features of the lobar bronchus. Since the bronchus of the right lower lobe of the lung is straighter and steeper than other bronchial branches, and the angle between the right lower lobe and the long axis of the trachea is smaller, the right lower lobar bronchus is more likely to be infected.

Previous studies have shown that the most common CT feature of COVID-19 pneumonia is the presence of multifocal GGOs [18, 19]. In this study, lesions were automatically classified by the uAI Intelligent Assistant Analysis System into GGOs, sub-solid lesions and solid lesions. Results showed that 2305 patients (94\%) presented primarily with GGO. This may be explained by the pathogenesis of the virus, with different stages of infection manifesting as a progression of abnormalities seen in the chest CT scans. As the disease progresses, alveolar septal capillaries dilate and become congested and alveolar exudates and lobular interstitial oedema appear. The CT scan would show patchy or flaky ground glass shadows. Local pulmonary vascular dilation demonstrated by dual-energy $\mathrm{CT}$ might accelerate the formation of GGO [20]. However, with an increase in alveolar exudate, expansion of intravascular interstitial blood vessels and aggravation of the alveolar and interstitial oedema, CT scans would progress to show a consolidation likened to ground glass. A gradual increase in the density of lesions may also be seen. The ground glass shadow then decreases in density or even completely disappears during the absorption period. Thus, even in the presence of changing lesion appearances, GGOs were present throughout all CT scans. The
Fig. 3 Anatomic distribution of infected bronchopulmonary segments in patients with COVID-19

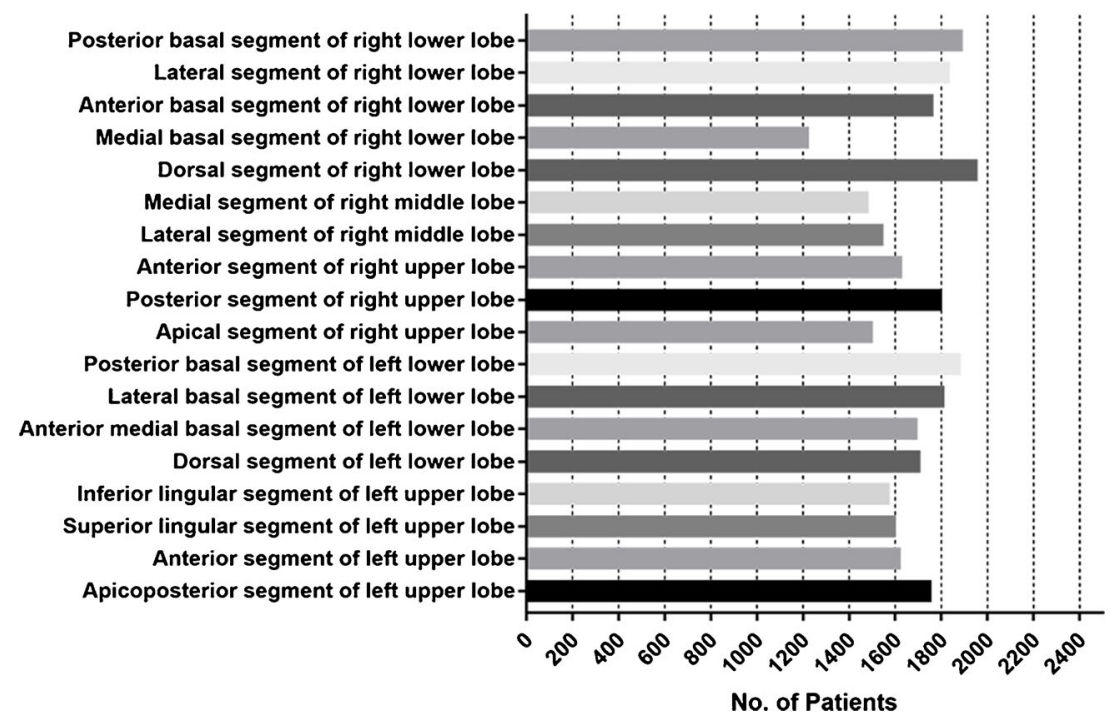


Table 3 Anatomic distribution of infected bronchopulmonary segments in patients with COVID-19

\begin{tabular}{|c|c|c|c|c|}
\hline \multirow{2}{*}{$\begin{array}{l}\text { Variable } \\
\text { Sex (reference: female) }\end{array}$} & \multirow{2}{*}{$\frac{\mathrm{OR}}{1.751}$} & \multicolumn{2}{|c|}{ OR $(95 \% \mathrm{CI})$} & \multirow{2}{*}{$\frac{p}{<0.001}$} \\
\hline & & 1.461 & 2.1 & \\
\hline Age (reference: under 60 years) & 4.907 & 4.092 & 5.884 & $<0.001$ \\
\hline \multicolumn{5}{|c|}{ Distribution (reference: medial basal segment of right lower lobe) } \\
\hline Apicoposterior segment of left upper lobe & 5.66 & 4.815 & 6.654 & $<0.001$ \\
\hline Anterior segment of left upper lobe & 3.58 & 3.055 & 4.194 & $<0.001$ \\
\hline Superior lingular segment of left upper lobe & 3.336 & 2.848 & 3.907 & $<0.001$ \\
\hline Inferior lingular segment of left upper lobe & 3.038 & 2.595 & 3.557 & $<0.001$ \\
\hline Dorsal segment of left lower lobe & 6.568 & 5.58 & 7.731 & $<0.001$ \\
\hline Anterior medial basal segment of left lower lobe & 4.58 & 3.902 & 5.376 & $<0.001$ \\
\hline Lateral basal segment of left lower lobe & 6.967 & 5.916 & 8.206 & $<0.001$ \\
\hline Posterior basal segment of left lower lobe & 9.126 & 7.727 & 10.779 & $<0.001$ \\
\hline Apical segment of right upper lob & 2.384 & 2.039 & 2.787 & $<0.001$ \\
\hline Posterior segment of right upper lobe & 6.69 & 5.682 & 7.876 & $<0.001$ \\
\hline Anterior segment of right upper lobe & 3.653 & 3.117 & 4.281 & $<0.001$ \\
\hline Lateral segment of right middle lobe & 2.779 & 2.375 & 3.252 & $<0.001$ \\
\hline Medial segment of right middle lobe & 2.242 & 1.918 & 2.62 & $<0.001$ \\
\hline Dorsal segment of right lower lobe & 12.301 & 10.377 & 14.582 & $<0.001$ \\
\hline Anterior basal segment of right lower lobe & 5.868 & 4.99 & 6.9 & $<0.001$ \\
\hline Lateral segment of right lower lobe & 7.621 & 6.465 & 8.984 & $<0.001$ \\
\hline Posterior basal segment of right lower lobe & 9.417 & 7.97 & 11.126 & $<0.001$ \\
\hline
\end{tabular}

study also found that sub-solid lesions were more likely to be found in the CT scans of elderly patients compared to those of younger patients. This may be related to the general observation of COVID-19 pneumonia being more serious in the elderly [21].

AI-based software has become one of the dominant approaches in fighting against COVID-19. The uAI Intelligent Assistant Analysis System integrates humanin-the-loop strategy into the training of a VB-net-based segmentation network, which involves interactivity with radiologists into the training of the network [13]. In addition, AI can improve work efficiency by accurate delineation of infections in CT images, facilitating subsequent quantification [14]. Moreover, assessing the disease by AI-based software is of great importance that could help decision making in treatment planning. Although there are still limited studies, we believe that the more accurate and objective data provided by intelligent software will greatly improve the quality of healthcare.

The present study had several limitations. Due to the hypoxemia and inability to move, many critically ill patients
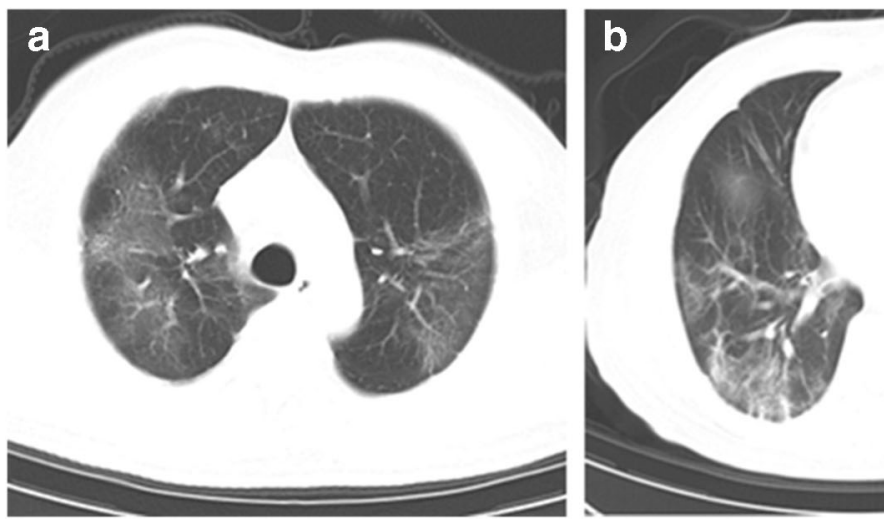

Fig. 4 Representative CT scans of the different lesions observed in patients with COVID-19 pneumonia. A A 52-year-old man. CT image showing ground glass opacity lesions in both the upper lung fields. B $A$

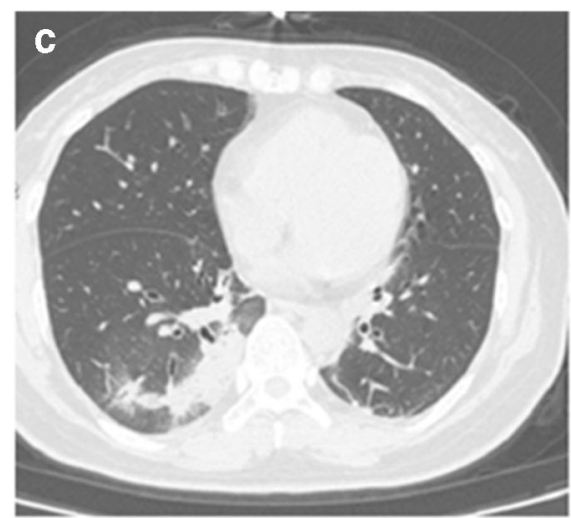

46-year-old woman. CT image showing ground glass opacity lesions mixed with sub-solid lesions in both the lower lung fields. C A 35-yearold woman. CT image showing solid lesions in the right lower lung fields 
Table 4 Distribution of CT signs in relation to age and sex

\begin{tabular}{lllll}
\hline Category & CT signs & & $P$ value \\
\cline { 2 - 4 } & Negative & $\begin{array}{l}\text { Primarily presenting with } \\
\text { GGO }\end{array}$ & $\begin{array}{l}\text { Primarily presenting with sub-solid } \\
\text { lesions }\end{array}$ & \\
\hline Sex & & & & 0.4125 \\
Male & $37(3 \%)$ & $1175(94 \%)$ & $38(3 \%)$ & \\
Female & $47(4 \%)$ & $1130(93 \%)$ & $33(3 \%)$ & $<0.001$ \\
Age & & & $22(2 \%)$ & \\
$<60$ years & $71(6 \%)$ & $1124(92 \%)$ & $49(4 \%)$ & \\
$\geq 60$ years & $13(1 \%)$ & $1181(95 \%)$ & &
\end{tabular}

Data presented as $n(\%)$

CT computed tomography cannot undergo CT examinations. Therefore, not all patients can benefit from this novel technology. Secondly, there was a need to adjust the uAI Intelligent Assistant Analysis System manually when identifying atypical lesions. Finally, since the study's data was only extracted from the baseline CT and one single-centre CT, there is a need for more in-depth research on evaluating the potential impact of AI software in COVID-19 patient management.

In conclusion, the use of chest $\mathrm{CT}$ combined with subsequent analysis by the UAI Intelligent Assistant Analysis System can accurately evaluate the pneumonia in COVID-19 patients. Typical CT features of COVID-19 pneumonia include multifocal bilateral GGOs, with the most common site of infection being the dorsal segment of the right lower lobe. The ability of the uAI Intelligent Assistant Analysis to quickly and accurately localize and quantify infection regions from CT scans will not only aid in the diagnosis of COVID-19, but also aid in assessing the disease to help guide physicians in their treatment plans.

Acknowledgements Thanks are due to Prof. Zhijun Tan for his assistance in statistical analysis.

Authors' contributions T.Z, X.J.Z and H.T.Z designed the study; H.T.Z, J.S.Z and H.H. $Z$ were major contributors in writing the manuscript; Y.D. N, Y. Z, E.Q. F, Y.H.X, W. L, W. P L, H. J Z, H. J, C.M.L, Y.Y. L, R.N.M, S.K.D and B.B. G collected the imaging and clinical data; all authors have read and approved the manuscript.

Funding information Key Research and Development Program of Shaanxi province (No. 2019SF-009).

\section{Compliance with ethical standards}

Conflict of interest The authors declare that they have no conflict of interest.

Ethics approval/consent to participate The study was conducted in accordance with the principles of the Declaration of Helsinki, and Institutional Review Board approval of Huoshenshan Hospital has been obtained. The written informed consent for this retrospective study was waived.

\section{References}

1. Zhu N, Zhang D, Wang W, Li X, Yang B, Song J, et al. A novel coronavirus from patients with pneumonia in China, 2019. N Engl J Med. 2020;382:727-33. https://doi.org/10.1056/NEJMoa2001017.

2. Organization WH. WHO Director-General's opening remarks at the media briefing on COVID-19 - 11. 2020. https://www.who.int/dg/ speeches/detail/who-director-general-s-opening-remarks-at-themedia-briefing-on-covid-19\%2D\%2D-11-march-2020. Accessed 13 July 2020

3. Organization WH. Coronavirus disease (COVID-2019) situation reports. https://covid19.who.int/. Accessed 13 July 2020

4. Jin YH, Cai L, Cheng ZS, Cheng H, Deng T, Fan YP, et al. A rapid advice guideline for the diagnosis and treatment of 2019 novel coronavirus (2019-nCoV) infected pneumonia (standard version). Mil Med Res. 2020;7:4. https://doi.org/10.1186/s40779-020-02336.

5. Zu ZY, Jiang MD, Xu PP, Chen W, Ni QQ, Lu GM, et al. Coronavirus disease 2019 (COVID-19): a perspective from China. Radiology. 2020:200490. https://doi.org/10.1148/radiol. 2020200490.

6. Hao W, Li M. Clinical diagnostic value of CT imaging in COVID19 with multiple negative RT-PCR testing. Travel Med Infect Dis. 2020:101627. https://doi.org/10.1016/j.tmaid.2020.101627.

7. Zhu Y, Liu YL, Li ZP, Kuang JY, Li XM, Yang YY, et al. Clinical and CT imaging features of 2019 novel coronavirus disease (COVID-19). J Infect. 2020. https://doi.org/10.1016/j.jinf.2020. 02.022 .

8. Li Y, Xia L. Coronavirus Disease 2019 (COVID-19): role of chest CT in diagnosis and management. AJR Am J Roentgenol. 2020:17. https://doi.org/10.2214/AJR.20.22954.

9. Bai HX, Hsieh B, Xiong Z, Halsey K, Choi JW, Tran TML, et al. Performance of radiologists in differentiating COVID-19 from viral pneumonia on chest CT. Radiology. 2020:200823. https://doi.org/ 10.1148/radiol.2020200823.

10. Li L, Qin L, Xu Z, Yin Y, Wang X, Kong B, et al. Artificial intelligence distinguishes COVID-19 from community acquired pneumonia on chest CT. Radiology. 2020:200905. https://doi.org/ 10.1148/radiol.2020200905.

11. Milletari F, Navab N, Ahmadi S-A. V-net: Fully convolutional neural networks for volumetric medical image segmentation. 2016 Fourth International Conference on 3D Vision (3DV); 2016 : IEEE; 2016. p. 565-71.

12. He K, Zhang X, Ren S, Sun J. Deep residual learning for image recognition. Proceedings of the IEEE conference on computer vision and pattern recognition; 2016; 2016. p. 770-8. 
13. Shan F, Gao Y, Wang J, Shi W, Shi N, Han M, et al. Lung infection quantification of COVID-19 in CT images with deep learning. arXiv e-prints. 2020. https://arxiv.org/abs/2003.04655. Accessed 13 July 2020

14. Shi F, Wang J, Shi J, Wu Z, Wang Q, Tang Z, et al. Review of artificial intelligence techniques in imaging data acquisition, segmentation and diagnosis for COVID-19. IEEE Rev Biomed Eng. 2020. https://doi.org/10.1109/RBME.2020.2987975.

15. Li D, Wang D, Dong J, Wang N, Huang H, Xu H, et al. Falsenegative results of real-time reverse-transcriptase polymerase chain reaction for severe acute respiratory syndrome coronavirus 2 : role of deep-learning-based CT diagnosis and insights from two cases. Korean J Radiol. 2020;21:505-8. https://doi.org/10.3348/kjr.2020. 0146.

16. Guan CS, Lv ZB, Yan S, Du YN, Chen H, Wei LG, et al. Imaging features of coronavirus disease 2019 (COVID-19): evaluation on thin-section CT. Acad Radiol. 2020. https://doi.org/10.1016/j.acra. 2020.03.002.

17. Ye Z, Zhang Y, Wang Y, Huang Z, Song B. Chest CT manifestations of new coronavirus disease 2019 (COVID-19): a pictorial review. Eur Radiol. 2020. https://doi.org/10.1007/s00330-02006801-0.

18. Zhou S, Wang Y, Zhu T, Xia L. CT features of coronavirus disease 2019 (COVID-19) pneumonia in 62 patients in Wuhan, China. AJR Am J Roentgenol. 2020:1-8. https://doi.org/10.2214/AJR.20. 22975.

19. Han R, Huang L, Jiang H, Dong J, Peng H, Zhang D. Early clinical and CT manifestations of coronavirus disease 2019 (COVID-19) pneumonia. AJR Am J Roentgenol. 2020:1-6. https://doi.org/10. 2214/AJR.20.22961.

20. Lang M, Som A, Mendoza DP, Flores EJ, Reid N, Carey D, et al. Hypoxaemia related to COVID-19: vascular and perfusion abnormalities on dual-energy CT. Lancet Infect Dis. 2020. https://doi. org/10.1016/S1473-3099(20)30367-4.

21. Liu K, Chen Y, Lin R, Han K. Clinical features of COVID-19 in elderly patients: a comparison with young and middle-aged patients. J Infect. 2020. https://doi.org/10.1016/j.jinf.2020.03.005.

Publisher's note Springer Nature remains neutral with regard to jurisdictional claims in published maps and institutional affiliations. 